Bio - grafía. Escritos sobre la Biología y su Enseñanza. ISSN 2027-1034

Edición Extraordinaria. p.p. 568 - 578

Memorias del IX Encuentro Nacional de Experiencias en Enseñanza de la Biología y la

Educación Ambiental. IV Congreso Nacional de Investigación en Enseñanza de la Biología.

\title{
I ENCUENTRO AMBIENTAL: UNIVERSIDAD, AMBIENTE Y SUSTENT ABILIDAD, EXPERIENCIAS Y PRÁCTICAS. APORTES ALA INCLUSIÓN DE LA DIMENSIÓN AMBIENTAL EN LA UPN
}

\author{
Adriana Tovar Martínez \\ Sonia Martínez
}

\section{RESUMEN}

La crisis de civilización de nuestra sociedad actual nos impone el reto de generar sendos esfuerzos en la construcción de un pensamiento y una cultura ambiental, la UPN como formadores de maestros viene realizando esfuerzos en la formación de ciudadanos capaces de tomar decisiones que propendan por el cuidado de la vida y la construcción de una sociedad responsable con el medio ambiente. Actualmente se vienen realizando esfuerzos en la Facultad de Ciencia y Tecnología. El objetivo de esta investigación fue identificar las formas de incorporación de la Dimensión Ambiental en la Facultad de Ciencia y Tecnología a través de la socialización de las prácticas y experiencias en el Primer Encuentro Ambiental: Ambiente y sustentabilidad, experiencias y prácticas. Se constituyó en un espacio de socialización de la investigación, la docencia y las prácticas pedagógicas. Este evento se realizó el día 20 de octubre del 2016, con un total de 300 personas (20 ponencias, 12 talleres y 50 posters, en las instalaciones de la Universidad Pedagógica Nacional. Encontramos una diversidad de enfoques y perspectivas que tienen lugar en las propuestas de trabajo, lo cual, se constituye en un referente y un potencial para pensar y fortalecer los procesos de incorporación de la Dimensión Ambiental en la FCT. Estos, elementos servirán de base para enriquecer los diálogos inter-departamentos e intrafacultad, como un primer paso en las acciones que demanda el trabajo académico para aportar en el desarrollo del eje 4. Universidad y Sustentabilidad Ambiental.

PALABRAS CLAVES: Educación Ambiental, dimensión ambiental, interdisciplinariedad, interculturalidad.

\section{ABSTRACT}

The crisis of civilization in our current society imposes us the challenge and efforts in the construction fo an environmental culture, the UPN as teachers of teachers has been making efforts in the formation of citizens capable of making decisions that tend to care of life and the construction of responsible societies with the environment. Currently, efforts are being made in the Faculty of Science and Technology (FCT). The objective of this research was to identify the ways of Environmental Dimension has been inluded in the Faculty of Science and Technology, through the socialization of practices and experiences in the First Environmental Meeting: Environment and sustainability, experiences and practices. This space allowed the socialization of research, teaching and pedagogical 
Bio - grafía. Escritos sobre la Biología y su Enseñanza. ISSN 2027-1034

Edición Extraordinaria. p.p. 568 - 578

Memorias del IX Encuentro Nacional de Experiencias en Enseñanza de la Biología y la

Educación Ambiental. IV Congreso Nacional de Investigación en Enseñanza de la Biología.

practices. This event was done on October 20, 2016, with a total of 300 people (20 papers, 12 workshops and 50 posters), in Universidad Pedagógica Nacional. We find a variety of approaches and perspectives that take place in the proposals of work, which is a reference and a potential to think and strengthen the processes of incorporation of the Environmental Dimension in the FCT. These elements will serve as a basis for enriching interdepartmental and intra-faculty dialogues as a first step in the actions that the academic work demands to contribute in the development of the axis 4. University and Environmental Sustainability.

KEY WORDS: Environmental Education, environmental dimension, interdisciplinarity, interculturality.

\section{INTRODUCCIÓN}

El mundo contemporáneo plantea importantes retos a las instituciones de educación superior latinoamericanas respecto al abordaje de la denominada crisis ambiental en la que subyace una crisis de civilización y en última instancia una crisis del conocimiento (Leff, 2014). En tal sentido se requiere de numerosos esfuerzos por parte de la sociedad y en especial de la universidad para aportar en la construcción de un pensamiento y una cultura ambiental, la formación ciudadana, y la toma de decisiones como parte de la responsabilidad socio-ambiental.

De igual forma, la Universidad Pedagógica Nacional en su plan de Desarrollo Institucional 2014-2019, consciente de su compromiso con la sociedad, el ambiente y la sustentabilidad, plantea el eje: Universidad y Sustentabilidad Ambiental, como oportunidad para incidir en la educación e historia del país, al ser la educadora de educadores que puede asumir un papel central en la transición hacia la configuración de sociedades sustentables, desde una mirada plural y compleja que demandan una lectura crítica y la transformación de realidades, de modo que se avance de una parte en la incorporación de la dimensión ambiental en la universidad y de otra, participe a través de redes y el trabajo articulado con otras instituciones en la educación ambiental del país. De acuerdo con lo anterior, es importante señalar los avances que en esta materia se registran en la Facultad de Ciencia y Tecnología, desde los Departamentos de Biología, Química y Física, en especial en los procesos de formación de profesores, así como, la apertura que se empieza a vislumbrar en los Departamentos de Matemáticas y Tecnología; asunto que invita a identificar las formas de incorporación de la dimensión ambiental y las perspectivas que se abordan. Estos, elementos servirán de base para enriquecer los diálogos inter-departamentos e intrafacultad, como un primer paso en las acciones que demanda el trabajo académico para aportar en el desarrollo del eje 4.Universidad y Sustentabilidad Ambiental.

El objetivo de esta investigación fue identificar las formas de incorporación de la Dimensión Ambiental en la Facultad de Ciencia y Tecnología a través de la socialización 
Bio - grafía. Escritos sobre la Biología y su Enseñanza. ISSN 2027-1034

Edición Extraordinaria. p.p. 568 - 578

Memorias del IX Encuentro Nacional de Experiencias en Enseñanza de la Biología y la

Educación Ambiental. IV Congreso Nacional de Investigación en Enseñanza de la Biología.

de las prácticas y experiencias en el Primer Encuentro Ambiental Universidad, Ambiente y Sustentabilidad.

\section{METODOLOGIA}

ORGANIZACIÓN DEL PRIMER ENCUENTRO AMBIENTAL: Universidad, Ambiente y Sustentabilidad. Experiencias y Prácticas.

La organización del Primer Encuentro Ambiental, fue producto de un trabajo pensado y articulado entre el equipo de proyecto de la Facultad; el Grupo de Investigación Educación en Ciencias, Ambiente y Diversidad y la vinculación del Grupo de Seminario de Educación Ambiental del Departamento de Biología. De esta forma, es importante señalar que la naturaleza y envergadura de esta propuesta requiere de un trabajo colectivo y colaborativo para poder lograr desarrollar en tan corto tiempo lo que exige este tipo de encuentros, entendiendo que es una oportunidad para visibilizar las experiencias y prácticas de maestros, estudiantes y egresados sobre ambiente $y$ sustentabilidad de la FCT. Así mismo, este encuentro también es motivo de sistematización por parte del equipo en colaboración con el grupo de seminario y de Investigación, de modo que permite documentar la experiencia.

El presente proyecto es de tipo cualitativo con un enfoque Descriptivo e interpretativo. En tal sentido, Vasilachis, (2013) plantea que la reflexión epistemológica en las ciencias sociales propende por liberarse de los dogmatismos de aquellas epistemologías que suponen una sola forma legítima de conocer, en tal sentido, advierte al investigador no cesar en los esfuerzos que realiza para superar los límites con los que se enfrenta día a día en su intento de conocer, lo que le da un carácter multidisciplinar, multiparadigmatico, entre otros. En consecuencia los diferentes participantes de la comunidad universitaria, tienen percepciones que en sí mismas son diversas, de acuerdo con sus experiencias y conocimientos, que permitirán contar con muchas ópticas y lecturas de lo ambiental y la sustentabilidad, en la UPN.

A partir de los hallazgos se realizó una categorización de las temáticas abordadas por los participantes, percepciones de sustentabilidad, enfoques investigativos, asociaciones al discurso ambiental, y lineamientos investigativos, que permitieron comprender la diversidad de iniciativas a través de los cuales los estudiantes y maestros de la FCT han venido incluyendo la dimensión ambiental desde sus campos de acción.

\section{RESULT ADOS Y DISCUSION}

El primer Encuentro Ambiental, se constituyó en un espacio de socialización de la investigación, la docencia y las prácticas pedagógicas relacionadas con el ambiente y la sustentabilidad que desarrollan profesores formadores de la Facultad, profesores en formación y egresados. En tal sentido, se resalta la destacada participación 
Bio - grafía. Escritos sobre la Biología y su Enseñanza. ISSN 2027-1034

Edición Extraordinaria. p.p. 568 - 578

Memorias del IX Encuentro Nacional de Experiencias en Enseñanza de la Biología y la Educación Ambiental. IV Congreso Nacional de Investigación en Enseñanza de la Biología.

con un total de 300 personas inscritas, para este evento académico realizado el día 20 de octubre del 2016, con una intensidad de 10 horas, en las instalaciones de la Universidad Pedagógica Nacional y las salas del Auditorio Gabriel Betancurt de la UPN.

Participación por Departamentos

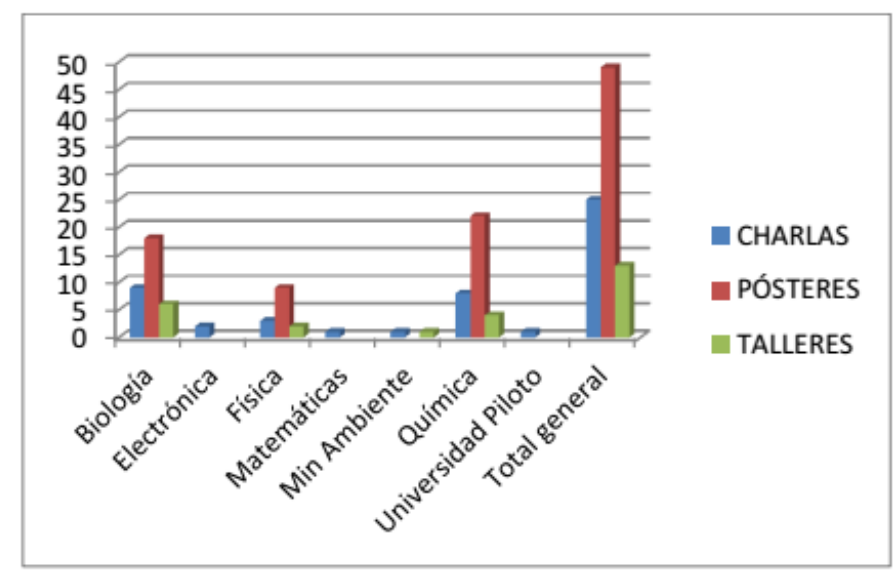

En relación con la participación de trabajos, se aprecia un amplio número por parte de los Departamentos de Química y Biología, seguidos por el Departamento de Física y en menor proporción los Departamentos de Tecnología y Matemáticas, así, el Programa Curricular en Química participo con un $41 \%$ en Posteres, registro consignado y analizado en una matriz, por estudiantes del Seminario de educación ambiental de profundización 2016.2 de Biología y dirigido por una profesora del DBI; Biología participo con un $19 \%$ en posteres, pero con $67 \%$ en talleres; Fisica con $25 \%$ en pósteres; la Maestría en Matemáticas con $13 \%$ y Tecnología en Electronica $3 \%$. En entrevistas realizadas a los profesores de la Facultad se encontró que, existe mayor énfasis curricular en los Departamento de Química, Biología y Física, y con menor énfasis en Tecnología y Matemáticas.

Además se registraron otras Universidades participantes como la Sede de Valle de Tensa de la UPN, la Universidad Distrital con profesores participantes en ponencia y Charla central; la Universidad Minuto de Dios con Charla y poster y la Universidad El Bosque, en donde los estudiantes realizaban las practicas pedagógicas de los diferentes programas curriculares de la Facultad, quienes mostraron sus avances en un alto porcentaje $58 \%$ a través de pósteres. 
Bio - grafía. Escritos sobre la Biología y su Enseñanza. ISSN 2027-1034

Edición Extraordinaria. p.p. 568 - 578

Memorias del IX Encuentro Nacional de Experiencias en Enseñanza de la Biología y la

Educación Ambiental. IV Congreso Nacional de Investigación en Enseñanza de la Biología.

Tipos de participación

\section{Tipo de Participación}

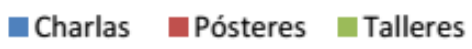

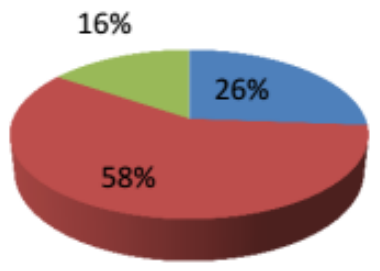

De manera que este fue un encuentro que contó con la participación de niños, jóvenes, profesores en formación inicial, profesores en ejercicio y profesores formadores, que permitieron generar un diálogo y un intercambio de experiencias y propuestas innovadoras además de encontrar en los procesos educativos un horizonte de sentido en la configuración de sociedades comprometidas con el ambiente desde prácticas de sustentabilidad. Así las diferentes perspectivas que presentaron abarcaron algunas corrientes como: en un $44 \%$ tenían que ver con aspectos socio-ambientales; otro $25 \%$ con los ecosistemas y sus interacciones con la biota; un $16 \%$ con los conceptos desde la ecología, linnología, los bioindicadores ambientales, entre otros; un $9 \%$ en aspectos sociopolíticos como los del estudio del asbesto en la parte de salud pública y toxicología, la química y la patología; el resto y no menos importantes los temas se relacionaron con justicia social al presentar los megaproyectos para la ciudad de Bogotá; biofísicos como el asunto del reciclaje de material sólido y lixiviados.

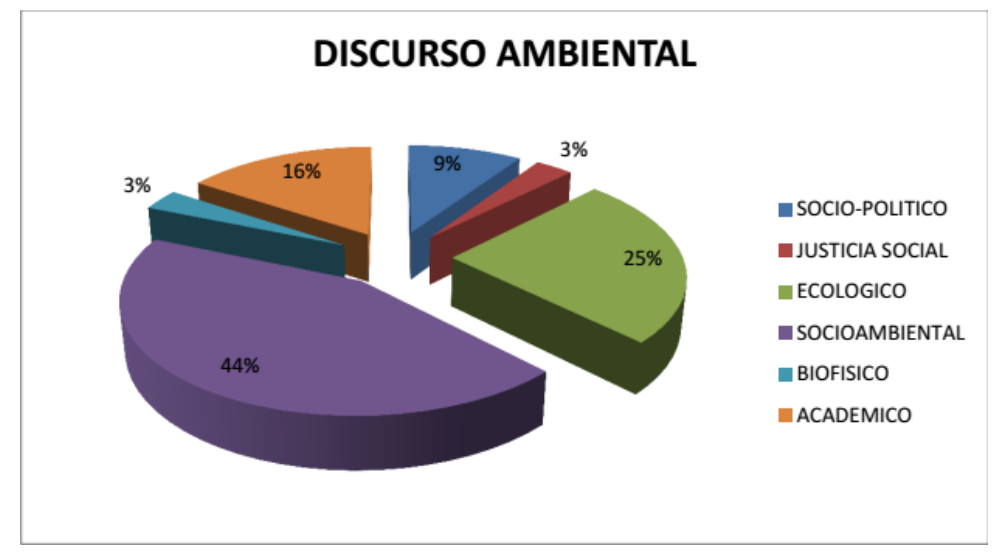

Las experiencias que tuvier on la modalidad de talleres fueron realizados por estudiantes y profesores especialistas y el Ministerio de Ambiente y Desarrollo Sostenible, por ejemplo el de "Plástica participativa para la apropiación del medio ambiente", quienes orientaron el 
Bio - grafía. Escritos sobre la Biología y su Enseñanza. ISSN 2027-1034

Edición Extraordinaria. p.p. 568 - 578

Memorias del IX Encuentro Nacional de Experiencias en Enseñanza de la Biología y la

Educación Ambiental. IV Congreso Nacional de Investigación en Enseñanza de la Biología.

dibujo de murales in situ del edificio $\mathrm{B}$, a través de una representación gráfica para la materialización de ideas, con una voluntad particular o colectiva, en su concreción. Otras experiencias presentadas en taller comprenden la "Danza del Agua", "Mariposas Andinas", "día mundial de la vida silvestre - Manos Unidas" y "Chilito un jardín hecho memoria", que señalan la importancia de considerar otras maneras de expresar el vínculo, propiciar un tejido de lo colectivo y materilizar a través del arte una mirada de lo ambiental.

Se observa que en relación con los diferentes trabajos socializados, predomina discurso socioambiental en la modalidad de póster con un $44 \%$, por lo cual se destaca, que el papel de los profesores de ciencias no solo obedece al desarrollo del cuerpo de contenidos disciplinares, sino que empiezan a considerar las discusiones en términos de las relaciones de las disciplinas con el contexto y con las realidades sociales que repercuten en algunos casos en problemáticas acuciantes para la comunidad educativa, y que requieren un tratamiento que permita establecer una mirada crítica, reflexiva, relacional y propositiva desde la acción educativa.

El discurso sociambiental sobre el cual se erige buena parte de las propuestas supone la necesidad de un modelo de educación que responda a los contextos locales y dialogue con el mundo global, en ese sentido Morín, (1998) afirma que la educación socioambiental fundada en una visión sistémica debe contemplar a los sujetos como un conjunto articulado cuya finalidad gira en torno a procesos de socialización/resocialización dirigidos a conseguir la adaptación, la integración y la emancipación de los individuos en la sociedad y que a su vez, estos objetivos educativos orientados al sujeto reviertan directamente en la mejora social, de modo que se propende potenciar las capacidades individuales y colectivas, que de igual forma contribuya en el cuidado y protección del ambiente. Por su parte, el discurso ecológico, emerge en el $25 \%$ de las propuestas al considerar necesario los procesos de conocimiento y valoración de la base eco sistémica, como los Programas de Biología, Química. Física y la maestría de Matemáticas, que permitan adelantar procesos de investigación y de educación hacia el fortalecimiento de estrategias que propicien en la comunidad educativa el conocimiento, apropiación y valoración de los ecosistemas desde un abordaje sistémico de manera que se avance en procesos de conservación y restauración de diversos ecosistemas tanto en los centros urbanos como en los contextos rurales. 
Bio - grafía. Escritos sobre la Biología y su Enseñanza. ISSN 2027-1034

\section{Edición Extraordinaria. p.p. 568 - 578}

Memorias del IX Encuentro Nacional de Experiencias en Enseñanza de la Biología y la Educación Ambiental. IV Congreso Nacional de Investigación en Enseñanza de la Biología.

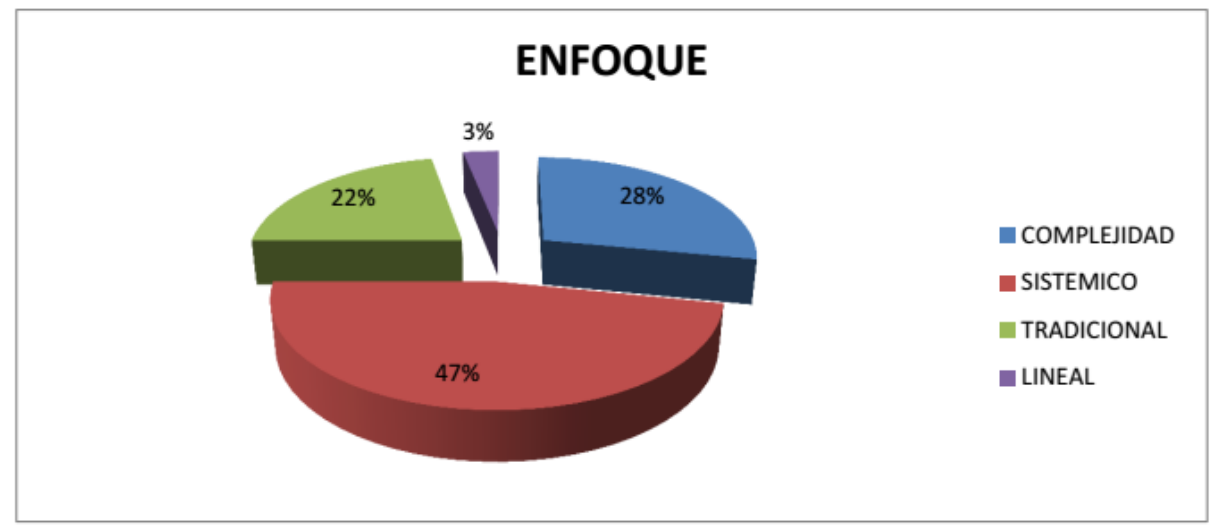

En cuanto al enfoque de los trabajos, se observa que un $47 \%$ acoge el enfoque sistémico, en el cual, resulta indispensable desarrollar propuestas teóricas y prácticas, que comprenden salidas de campo, para interpretar los sistemas ambientales de estudio y de otra parte, trabajar en terreno con las comunidades, de modo que se pueda hacer una interpretación del sistema, reconocer las problemáticas de modo que de acuerdo con Sauvé, se pretende conocer y comprender adecuadamente las realidades y las problemáticas ambientales desde un énfasis ecológico, con un trabajo que destaca la interrelación de los seres humanos y el medio natural.

En esta misma categoría, se aprecia que un $28 \%$ de las propuestas se enfocan desde la complejidad, al plantear una ruptura con una visión de mundo que se funda en la relación causa-efecto de la ciencia clásica, para hacer una lectura de las realidades ambientales, que de acuerdo con Morín (1998) comprende un modo de pensamiento el cual, "respeta la multidimensionalidad, la riqueza, el misterio de lo real y que las determinaciones cerebral, cultural, social, histórica que experimenta todo pensamiento co-determinan siempre el objeto de conocimiento". De modo, que este enfoque constituye un abordaje que apela a comprender lo ambiental desde una mirada compleja que exige un posicionamiento diferente por parte del investigador y educador para asumir las realidades y propiciar alternativas de un ambiente sistémico, complejo y cambiante.

Llama la atención que en estas propuestas se empieza a dar apertura a otras formas de conocimiento y en tal sentido se expresa un reconocimiento de los saberes locales, que forman parte de las experiencias de los sujetos y colectivos con el ambiente en el que viven, así, se considera la necesidad de posibilitar espacios para el diálogo que permitan desde las diferentes formas de conocimiento plantear alternativas de solución a problemáticas ambientales, que requieren no solo de un conocimiento unidireccional, sino por el contrario ampliar los marcos explicativos y las propuestas desde perspectivas plurales que viabilicen una mayor apropiación y participación de las comunidades directamente implicadas. 
Bio - grafía. Escritos sobre la Biología y su Enseñanza. ISSN 2027-1034

Edición Extraordinaria. p.p. 568 - 578

Memorias del IX Encuentro Nacional de Experiencias en Enseñanza de la Biología y la

Educación Ambiental. IV Congreso Nacional de Investigación en Enseñanza de la Biología.

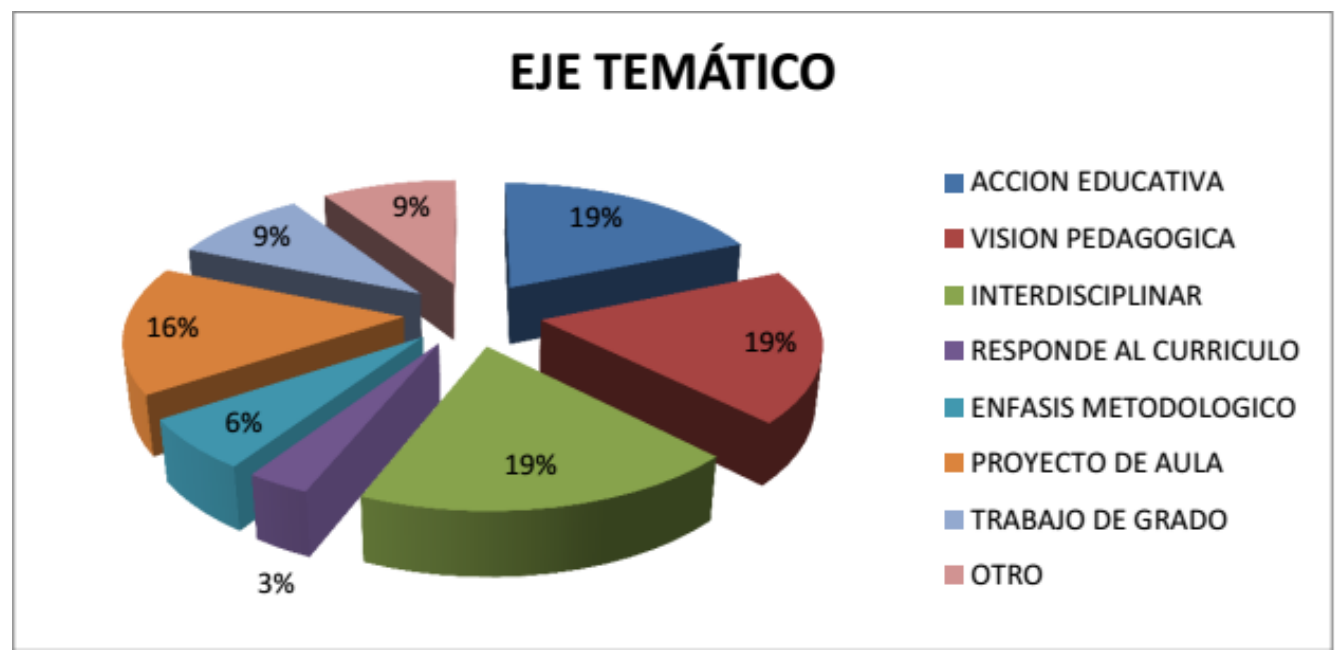

De acuerdo con los resultados obtenidos, la categoría Eje temático, ofrece una diversidad de abordajes, en los que se encuentra que la mayor parte de los trabajos con el más alto porcentaje corresponde a las subcategorías Acción Educativa con un 19\%. Cabe resaltar, que buena parte de las experiencias de aula y de la acción pedagógica, comprende proyectos y propuestas de profesores formadores y de profesores en ejercicio que adelantan estudios de maestría en la Facultad de ciencia y Tecnología, señalando la importancia e incidencia que tienen los procesos de formación tanto en el currículo de algunos programas de la universidad como en los proyectos y trabajos en las instituciones educativas de la básica y media.

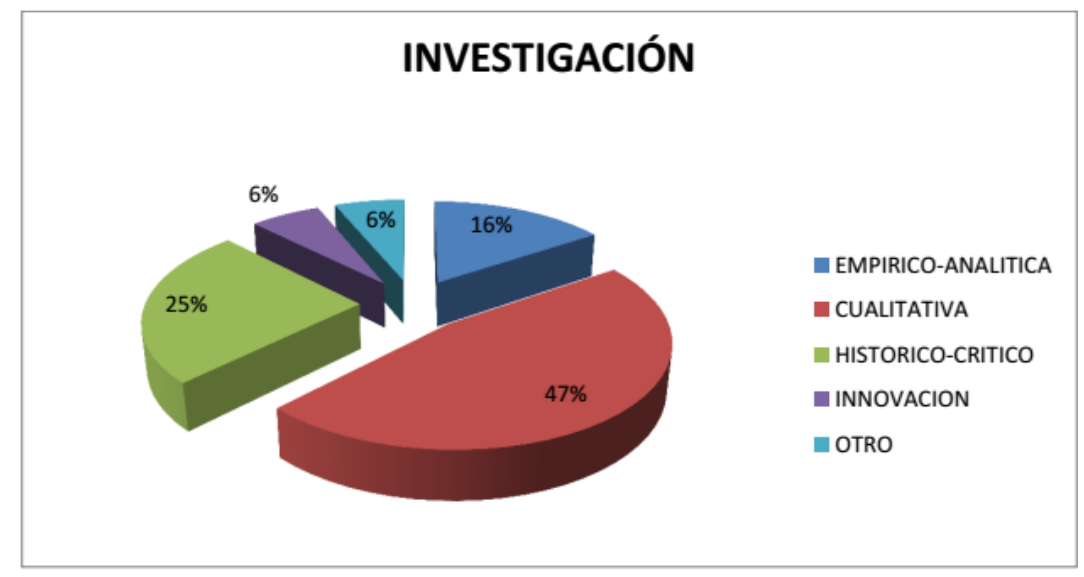

En la gráfica correspondiente a la categoría de Investigación se puede observar que el $47 \%$ de los trabajos, corresponden a proyectos de investigación que fundamentados en 
Bio - grafía. Escritos sobre la Biología y su Enseñanza. ISSN 2027-1034

Edición Extraordinaria. p.p. 568 - 578

Memorias del IX Encuentro Nacional de Experiencias en Enseñanza de la Biología y la

Educación Ambiental. IV Congreso Nacional de Investigación en Enseñanza de la Biología.

una metodología cualitativa, en tal sentido, es posible apreciar que se presenta un trabajo amplio y que la mayoría de propuestas se abordan con y desde las comunidades educativas o grupos particulares para hacer una lectura e interpretaciones de cuestiones socio-ambientales o socio-científicas, entre otras, con los que se adelanta la investigación. De otra parte, un $25 \%$ de los trabajos se realizaron con una metodología investigativa de tipo histórico-crítico, de modo que se legitima la necesidad de comprender las configuraciones de problemáticas ambientales en contexto y de hacer una lectura crítica de las mismas, como una manera que permite aportar alternativas para la toma de decisiones; así mismo, otro 16\% comprende un abordaje de tipo empírico-analítico, como especialmente se pudo apreciar en algunas de las propuestas de los Departamentos de Química y Física.

\section{Perspectivas de Sustentabilidad}

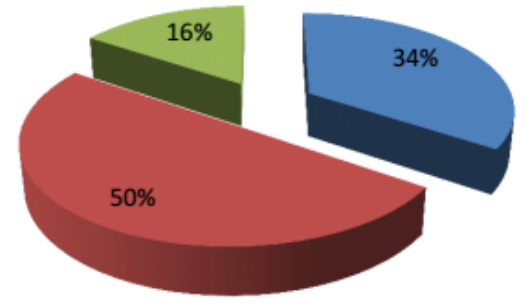

- SUSTENTABLE

- SOSTENIBLE

\#OTRO

En relación con la categoría Perspectivas de la Sustentabilidad se destaca con un $50 \%$ su abordaje en la mayoría de las propuestas educativas y proyectos de investigación, de modo que permiten apreciar su apropiación en los procesos educativos en los cuales supone una responsabilidad ambiental en beneficio de todos para mejorar las relaciones de los seres humanos tanto en lo individual como en lo colectivo con el medio. En varias de las propuestas se señala la necesidad de transformar las prácticas y generar acciones educativas que permitan el compromiso ciudadano para construir un ambiente sustentable. De otra parte, el $34 \%$ de los trabajos incorporan aspectos de la Educación para el Desarrollo Sostenible, lo cual obedece en parte por la década de la EDS 20152014, propuesta por la UNESCO y en la que Colombia asumió un compromiso a este respecto, de modo que ha influenciado en cierta medida los proyectos y trabajos en el ámbito investigativo y educativo, especialmente en los desarrollo en las instituciones educativas de la educación Básica y media, como parte de las acciones que se adelantan en los proyectos ambientales escolares y en las propuestas de innovación.

\section{CONCLUSIONES}

La responsabilidad que le asiste a la universidad comprende una reflexión crítica y propositiva de los procesos de Incorporación de la Dimensión Ambiental que demandan 


\title{
Bio - grafía. Escritos sobre la Biología y su Enseñanza. ISSN 2027-1034
}

\section{Edición Extraordinaria. p.p. 568 - 578}

\author{
Memorias del IX Encuentro Nacional de Experiencias en Enseñanza de la Biología y la \\ Educación Ambiental. IV Congreso Nacional de Investigación en Enseñanza de la \\ Biología.
}

nuevos e importantes desafíos en la formación docente y la investigación, de manera que se pretende desde un diálogo con los diferentes actores sociales aportar algunas recomendaciones para la Facultad de Ciencia y Tecnología, que permitan transitar y fortalecer este proceso. El I Encuentro Ambiental.

Dentro de los aspectos que emergen en el Primer Encuentro Ambiental, es importante señalar la diversidad de enfoques $y$ perspectivas que tienen lugar en las propuestas de trabajo, lo cual, se constituye en un referente y un potencial para pensar y fortalecer los procesos de incorporación de la Dimensión Ambiental en la FCT.

El mundo contemporáneo, presenta una serie de retos en relación a la complejidad ambiental y las problemáticas que se han agudizado en las últimas décadas tanto en términos de destrucción de ecosistemas, como aumento de la contaminación, la pérdida de la biodiversidad, la marginación de grupos minoritarios y aumento de la pobreza, que interpelan un estilo particular de conocer y relacionarse con el mundo, de manera que implican una mirada crítica de los sistemas educativos y de aquellas investigación alejadas de los contextos educativos y realidades ambientales. Aspectos que a su vez, requieren pensar en los procesos de formación, acción y participación en la construcción social del ambiente y la sustentabilidad, la convivencia, la alteridad que implican repensar la educación y la formación de profesores de cara al siglo XXI.

La formación ambiental en la educación superior plantea el desarrollo de competencias para la sustentabilidad desde enfoques de la complejidad y de perspectivas socio-críticas, que promuevan la participación y la toma de decisiones en la construcción de ambientes sustentables, fundadas en una equidad intra e intergeneracional, que apelan a la convivencia, la alteridad y el valor en sí mismo de la naturaleza frente a la permanencia de las diferentes formas de vida y valores asociados con la justicia social. De manera que la sustentabilidad ambiental comprende la articulación de las relaciones entre la naturaleza y lo social.

Es importante señalar que el trabajo con estudiantes y profesores de los diferentes programas de licenciatura de la Facultad de ciencia y Tecnología resultó muy enriquecedor al permitir desde sus experiencias personales aportar sus visiones de la sustentabilidad, así como, aportar alternativas para incorporar la dimensión ambiental en los programas de formación profesoral. Se destaca la mirada propositiva de los estudiantes y profesores participantes quienes expresan la posibilidad de adelantar un trabajo inter y tras-disciplinar, tanto en los currículos, como en las propuestas de investigación, que ofrecen un panorama amplio y posible de repensar las fronteras disciplinares para abordar una mirada compleja de la realidad, propuestas en las que confluyeron la mayoría de los futuros licenciados, no en vano aparece la importancia de formar en una ética que considera las diversidades, la alteridad, la reciprocidad, el cuidado del ambiente y de la conciencia ciudadana. En tal sentido, implica empezar a considerar procesos de trasformación en los ámbitos académicos e investigativos de la universidad, ante los nuevos retos del país en el mundo contemporáneo. 
Bio - grafía. Escritos sobre la Biología y su Enseñanza. ISSN 2027-1034

Edición Extraordinaria. p.p. 568 - 578

Memorias del IX Encuentro Nacional de Experiencias en Enseñanza de la Biología y la Educación Ambiental. IV Congreso Nacional de Investigación en Enseñanza de la Biología.

En tal sentido, uno de los desafíos en la incorporación de la dimensión ambiental es la formación integral de profesores en la investigación y formulación de propuestas educativas que permitan la articulación de los conocimientos disciplinares e interdisciplinares, la lectura de los contextos locales, el análisis de las problemáticas y la capacidad de plantear alternativas de solución, por tanto, dadas las perspectivas, experiencias y prácticas que señalan la diversidad que alberga la Facultad en el desarrollo de sus programas se plantea:

- Incorporar un seminario que contemple temáticas ambientales y de sustentabilidad en programas que en su plan de estudios aún no cuentan con un abordaje explícito en este sentido.

- Fortalecer un seminario electivo transversal a todos los programas de la Facultad que incorpore aspectos de la ciencia, la tecnología, la sociedad y el ambiente.

- Desarrollar prácticas pedagógicas que aborden la Dimensión Ambiental.

- Introducir experiencias de salidas de campo tanto en los programas como en espacios que sean transversales y que permitan una mirada interdisciplinar de los participantes de la FCT.

- La incorporación de contenidos ambientales y de sustentabilidad en los seminarios existentes como lo propuesto por profesores y estudiantes del Departamento de Matemáticas.

\section{BIBLIOGRAFÍA}

Leff, E. (2008). Discursos sustentables. México. Recuperado de: http://www.deliberaweb.com/dades/documents/497/1272914999.pdf

Morín, E. (1998). El pensamiento complejo. Entrevista realizada en Santafé de Bogotá el 5 de marzo de 1997 por Fabio Giraldo y José Malavar; Revista Ensayo \& Error; Santafé de Bogotá; Año 3, No.4, pp. 96 -113

Vasilachis de Gialdino, I. (2013). Discurso Científico, político, Jurídico, y de Resistencia. Análisis Lingüístico e Investigación Cualitativa. Barcelona: Gedisa 